\title{
A dynamic periplasmic electron transfer network enables respiratory flexibility beyond a thermodynamic regulatory regime
}

\author{
Gunnar Sturm ${ }^{1}$, Katrin Richter ${ }^{1}$, Andreas Doetsch ${ }^{2}$, Heinrich Heide ${ }^{3,5}$, Ricardo O Louro ${ }^{4}$ \\ and Johannes Gescher ${ }^{1}$ \\ ${ }^{1}$ Department of Applied Biology, Institute for Applied Biosciences, Karlsruhe Institute of Technology (CS), \\ Karlsruhe, Germany; ${ }^{2}$ Department of Microbiology of Natural and Technical Interfaces, Institute of \\ Functional Interfaces, Karlsruhe Institute of Technology (CN), Eggenstein-Leopoldshafen, Germany; \\ ${ }^{3}$ Department of Biochemistry, Institute for Biology II, University of Freiburg, Schänzlestr. 1, Freiburg, \\ Germany and ${ }^{4}$ Instituto de Tecnologica Química e Biológica, Universidade Nova de Lisboa, \\ Av. Da República - EAN, Oeiras, Portugal
}

\begin{abstract}
Microorganisms show an astonishing versatility in energy metabolism. They can use a variety of different catabolic electron acceptors, but they use them according to a thermodynamic hierarchy, which is determined by the redox potential of the available electron acceptors. This hierarchy is reflected by a regulatory machinery that leads to the production of respiratory chains in dependence of the availability of the corresponding electron acceptors. In this study, we showed that the $\gamma$-proteobacterium Shewanella oneidensis produces several functional electron transfer chains simultaneously. Furthermore, these chains are interconnected, most likely with the aid of $c$-type cytochromes. The cytochrome pool of a single S. oneidensis cell consists of ca. 700000 hemes, which are reduced in the absence on an electron acceptor, but can be reoxidized in the presence of a variety of electron acceptors, irrespective of prior growth conditions. The small tetraheme cytochrome (STC) and the soluble heme and flavin containing fumarate reductase FccA have overlapping activity and appear to be important for this electron transfer network. Double deletion mutants showed either delayed growth or no growth with ferric iron, nitrate, dimethyl sulfoxide or fumarate as electron acceptor. We propose that an electron transfer machinery that is produced irrespective of a thermodynamic hierarchy not only enables the organism to quickly release catabolic electrons to a variety of environmental electron acceptors, but also offers a fitness benefit in redox-stratified environments.
\end{abstract}

The ISME Journal (2015) 9, 1802-1811; doi:10.1038/ismej.2014.264; published online 30 January 2015

\section{Introduction}

Bacteria and Archaea have adapted to most of the environmental niches on the planet. To a large extent, their success is due to a physiological flexibility that enables them to thrive by choosing from a multitude of energy-generating metabolic strategies. Evolution has selected for a regulatory pattern described as a thermodynamic hierarchy, meaning that an organism with a certain metabolic repertoire will not simply choose a form of energy metabolism that is possible under given environmental conditions, but that will release the highest

Correspondence: J Gescher, Applied Biology, Karlsruhe Institute of Technology, Fritz Haber Weg 2, Geb. 30.44, Karlsruhe 76131, Germany.

E-mail: Johannes.gescher@kit.edu

${ }^{5}$ Current address: Thermo Fisher Scientific GmbH, Im Steingrund 4-6, 63303 Dreieich, Germany.

Received 2 July 2014; revised 24 November 2014; accepted 5 December 2014; published online 30 January 2015 amount of energy per mol of substrate consumed. In other words, organisms favor respiration over fermentation and respiratory electron acceptors with high rather than low redox potentials (Goh et al., 2005). Escherichia coli is an example of an organism with a very structured metabolism in which regulatory elements such as ArcAB or FNR direct metabolism according to the potential energy output of a reaction (Unden and Duchene, 1987; Spiro and Guest, 1990; Green and Guest, 1993).

Like E. coli, Shewanella oneidensis is a $\gamma$-proteobacterium, and it is the most versatile microbe known in terms of electron acceptors it can use. Thus far, it is the best understood model organism regarding the reduction of extracellular electron acceptors such as ferric iron or manganese oxides (Fredrickson et al., 2008; Richardson et al., 2012; Breuer et al., 2014). Interestingly, most electron acceptors are reduced by $S$. oneidensis either in the periplasm or at the cell surface. Moreover, the majority of pathways are dependent on the catalysis 
of electron transfer into the periplasm by the menaquinol oxidase and tetraheme cytochrome CymA (Marritt et al., 2012). Unsurprisingly, a cymA-deficient mutant shows growth deficiencies with a variety of anoxic electron acceptors (Myers and Myers, 1997). Although it was shown experimentally in vivo and in vitro that CymA can directly transfer electrons to the respiratory fumarate reductase FccA, it was suggested that a direct transfer would also be in place toward the periplasmic nitrate and nitrite reductases NapAB and NrfA, respectively (Schwalb et al., 2002; Gao et al., 2009; Schuetz et al., 2009).

Electron transfer to insoluble and/or membrane impermeable electron acceptors depends on multimeric protein complexes that are localized to the outer membrane. An example is the MtrABC complex involved in the reduction of insoluble metals as well as shuttling compounds. It consists of one cytochrome each on the periplasmic (MtrA) and cell surface (MtrC) site of the outer membrane, with a membrane-associated $\beta$-barrel protein $(\mathrm{MtrB})$ most likely acting as a connector between them (Ross et al., 2007; Shi et al., 2007; Hartshorne et al., 2009). Interestingly, the periplasmic space of $S$. oneidensis is with 235 A too wide for electron transfer to proceed directly between CymA and the periplasmic components of the above-mentioned multimeric complexes like MtrA. Therefore, it has to be assumed that proteins like MtrA can diffuse through the periplasm or that additional redox proteins mediate electron transfer through the periplasm. The genome of $S$. oneidensis MR-1 contains the genetic information for $27 c$-type cytochromes that are putatively localized to the periplasm, and a surprising number of them is expressed in high concentration immediately after conditions change from oxic to suboxic (Beliaev et al., 2005; Romine et al., 2008; Gao et al., 2010). Nevertheless, it is not known which of these cytochromes are necessary to bridge the periplasmic gap between CymA and the outer membrane. Interestingly, the fumarate reductase FccA and the small tetraheme cytochrome (STC) (CctA) are highly abundant in the periplasm under anoxic conditions (Tsapin et al., 2001; Meyer et al., 2004). Nevertheless, although it was suggested that they might have a role in the transfer of electrons to the outer membrane, a corresponding phenotype under conditions of ferric iron or dimethyl sulfoxide (DMSO) reduction was not shown so far (Gordon et al., 2000; Schuetz et al., 2009; Fonseca et al., 2013).

Our experiments showed that $S$. oneidensis does not appear to behave according to a thermodynamic hierarchy. Different respiratory chains are coexpressed simultaneously and comprise a dynamic electron transfer network. The $c$-type cytochromes seem to function as a buffering system for catabolic electrons, which can release its electron cargo to different electron acceptors, mostly irrespective of prior growth conditions. Moreover, STC and FccA seem to function as an electron transfer hub and share, in this regard, a functional redundancy. Single mutants of either of these two cytochromes show only minor phenotypic changes, if at all. A double mutant is unable to grow or strongly affected in growth with a variety of electron acceptors, but can be rescued with either of the two cytochromes.

\section{Materials and methods}

Bacterial strains and culture conditions

The strains used in this study are listed in Supplementary Table S1. Cultures of S. oneidensis were grown aerobically in lysogeny broth or M4 minimal medium with lactate $(50 \mathrm{~mm})$ as described by Schicklberger et al. (2013). For anaerobic growth, M4 minimal medium was supplemented with ferric citrate (50 mM), DMSO (50 mM), anthraquinone2,6-disulfonate $(10 \mathrm{~mm})$, trimethylamine $N$-oxide (TMAO) (25 mM), sodium nitrate $(2 \mathrm{~mm})$, sodium thiosulfate $(50 \mathrm{~mm})$ or fumarate $(50 \mathrm{~mm})$ as terminal electron acceptor (TEA) lactate as electron donor either in a concentration of $50 \mathrm{~mm}$ or in varying concentrations that stoichiometrically balanced the electron uptake capacity of the above-mentioned electron acceptors in the listed concentrations. Growth experiments were carried out in at least three independent replicates. Error bars represent s.d.

\section{Microscopic imaging}

Environmental scanning electron microscopy imaging was performed by mounting $S$. oneidensis cells, grown in M4 with ferric citrate as the TEA, onto the specimen holder. At a constant temperature of $3{ }^{\circ} \mathrm{C}$, a vacuum was slowly applied until the chamber pressure reached $519 \mathrm{~Pa}$.

\section{Cell fractionation}

The preparation of periplasmic and membrane fractions was performed as described by Schuetz et al. (2009). The expression of the $C$-type cytochromes was monitored via SDS-PAGE coupled with a heme-linked peroxidase stain as previously described (Thomas et al., 1976).

\section{Mass spectrometric analysis}

Periplasmic and membrane fractions of ferric irongrown $S$. oneidensis cells were analyzed using multidimensional protein identification technology as described by Schicklberger et al. (2011). Excised protein bands were analyzed using mass spectrometry according to Schuetz et al. (2009).

\section{Determination of hemes}

The heme content of the membrane and periplasmic fractions was determined using the method described by Berry and Trumpower (1987). A step-wise addition of sodium dithionite resulted in the 
successive reduction of hemes. Photometrical scans $(400-600 \mathrm{~nm})$ were recorded, and the first completely reduced spectrum was used for quantification. The absorption coefficient of $24 \mathrm{~mm}^{-1}$ (Berry and Trumpower, 1987) was applied using absorption values calculated according to equation 1 :

$$
A=(A 550(\text { red })-A 550(\text { ox }))-(A 535(\text { red })-A 535(\text { ox }))
$$

Calculation of the periplasmic space

It was possible to estimate the periplasmic volume by approximating the cellular shape as a cylinder covered by hemispheres. Based on this shape, the mathematical calculation of the periplasmic space was achieved using equation 2 :

$$
V=\left(\frac{4}{3} \pi\left(\frac{d}{2}\right)^{3}+\pi\left(\frac{d}{2}\right)^{2}(1-d)\right)-\left(\frac{4}{3} \pi\left(\frac{d}{2}-w\right)^{3}+\pi\left(\frac{d}{2}-w\right)^{2}(1-d)\right)
$$

where $V$ is the periplasmic volume, $l$ is the overall cell length, $d$ is the overall cell width, and $w$ is the periplasmic width. All values calculated are given with s.d.

\section{Cell counting}

Cell numbers were determined using a Neubauerimproved counting chamber with a $0.01-\mathrm{mm}$ depth. Prior to counting, the cells were fixed by the addition of 1 volume of $37 \%$ formaldehyde and incubated at $4{ }^{\circ} \mathrm{C}$ for $1 \mathrm{~h}$.

\section{Determination of the cellular redox state}

Cells were anaerobically grown in M4 medium with ferric citrate as TEA. The cells were harvested anoxically during the logarithmic growth phase and washed twice with M4 medium supplemented with anoxic lactate $(50 \mathrm{~mm})$ and lacking a TEA. Chloramphenicol in a concentration of $10 \mu \mathrm{g} \mathrm{ml}^{-1}$ was added to inhibit further protein synthesis. The cells were then resuspended in the same buffer and incubated at room temperature to allow for the complete reduction of the cells. Lactate was removed by washing the cells five times in M4 medium without lactate and a TEA, after which the cells were resuspended to an $\mathrm{OD}_{600}$ of 1.0 in the same medium. The reduced cells $(750 \mu \mathrm{l})$ were mixed with $250 \mu \mathrm{l}$ of the respective TEAs $(10 \mathrm{~mm}$ in M4), resulting in a final concentration of $2.5 \mathrm{~mm}$ TEA. Photometrical spectra $(400-600 \mathrm{~nm})$ were recorded immediately ( $8 \mathrm{~s}$ after addition of the electron acceptor) in an anoxic glove box using standard plastic cuvettes and a Tecan Infinite M200pro plate reader equipped with a cuvette slot. The cellular redox state was compared with a negative control to which TEA-free buffer had been added.
Construction of markerless S. oneidensis deletion mutants

Markerless deletion mutants were constructed as previously described (Schuetz et al., 2009). The pMQ150-based suicide vectors were assembled using the isothermal in vitro recombination method described by Gibson et al. (2009). Primers 1-4 (Supplementary Table S2) were used to construct a pMQ150-based cctA deletion vector, which was utilized as previously described (Shanks et al., 2006).

Cloning and expression of cctA and $f_{C C} A$

Primers $5+6$ and $7+8$ were used to amplify $\operatorname{cct} A$ and $n r f A$, respectively, with regions overlapping the pBAD202 plasmid. The fusion constructs were also cloned using isothermal in vitro recombination (Gibson et al., 2009). The resulting plasmids and the already available $f_{C C A}$ expression vector (Schuetz et al., 2009) were transformed into JG684 (Supplementary Table S1) by electroporation. Expression of genes was induced by the addition of $50 \mu \mathrm{M}$ arabinose. Arabinose was added to all cultures to provide similar growth conditions throughout the experiments.

Determination of $\mathrm{NO}_{3}^{-}$and $\mathrm{NO}_{2}^{-}$reduction

Cells were grown aerobically in M4 medium to an $\mathrm{OD}_{600}$ of 0.35 , harvested, and washed twice anoxically with M4 medium. Cell suspensions were made with an $\mathrm{OD}_{600}$ of 0.2 , and anaerobic $\mathrm{NO}_{3}^{-}$or $\mathrm{NO}_{2}^{-}$ reduction was initiated by the addition of the respective TEAs. Thus, $2 \mathrm{~mm} \mathrm{NO} \mathrm{NO}_{3}^{-}$or $\mathrm{NO}_{2}^{-}$was added to the cell suspension, and samples were collected every $15 \mathrm{~min}$. Further reduction was stopped by the addition of formic acid. The $\mathrm{NO}_{3}^{-}$ and $\mathrm{NO}_{2}^{-}$concentrations were measured using $\mathrm{NO}_{3}^{-}$ and $\mathrm{NO}_{2}^{-}$cuvette tests supplied by Hach-Lange (Berlin, Germany) as described by the manufacturer.

\section{Single-nucleotide resolution RNA sequencing}

Total RNA was prepared using an RNeasy kit provided by Qiagen (Hilden, Germany), and residual DNA was removed with the Ambion DNA-free kit (Life Technologies, Carlsbad, CA, USA). An Ambion MICROBExpress bacterial mRNA enrichment kit (Life Technologies) was used for ribosomal RNA depletion. Strand-specific cDNA libraries were prepared from $50 \mathrm{ng}$ of ribosomal RNA-depleted RNA following the TruSeq RNA protocol (Illumina, San Diego, CA, USA, without purification) with modification of the 2nd strand cDNA synthesis as previously described (Parkhomchuk et al., 2009). The libraries were prepared using multiplex primers to allow simultaneous sequencing in a single lane. Sequencing was performed on a HiSeq1000 using SBS v3 kits (Illumina) to generate paired-end reads of $2 \times 50$ nucleotides. Cluster detection and base calling were performed using RTA v1.13 (Illumina), 
and the quality of the reads was assessed with CASAVA v1.8.1 (Illumina). Sequencing was yielded between 17.7 and 53.2 million read pairs for each library with an average of $96.6 \%$ of the bases having a quality of Q30 or better.

\section{$R N A$-sequence data analysis}

The sequence data were mapped against the genome sequences of $S$. oneidensis MR-1 (NCBI accession number NC_004347.2) and its megaplasmid (NC_004349.1) using Bowtie2 (Langmead and Salzberg, 2012). Gene expression was calculated as reads per gene by determining the number of reads that overlapped with the annotated gene loci as previously described (Doetsch et al., 2012). The normalization of gene expression and statistical testing using a negative binomial distribution were performed using the R package 'DESeq' (Anders and Huber, 2010). Heatmaps of gene expression were generated with the 'heatmap.2' function of the $\mathrm{R}$ package 'gplots' (Warnes et al., 2013).

\section{Results}

Characterization of the periplasm as a cytochromebased electron transfer environment

This study aimed to elucidate the principles of periplasmic electron transfer. Therefore, the volume of the periplasm of a single cell was calculated to later estimate the average concentration of $c$-type cytochromes. The average length and width of $S$. oneidensis cells were measured using environmental scanning electron microscopy images of logarithmically growing ferric iron-reducing cells, and the width of the periplasm was obtained from the work of Dohnalkova et al. (2011). Using equation 2, these values led to an average periplasmic volume of $0.153 \mathrm{fl}$ per cell (Supplementary Figure S1). Furthermore, the concentration of heme cofactors in soluble and membrane fractions was quantified using absorption spectroscopy. As the heme cofactor is connected to the apoprotein in the periplasm, it was possible to directly calculate a concentration of soluble heme in the periplasm using the $0.153 \mathrm{fl}$ volume and the average number of total soluble heme sites per cell, which was quantified as 346142 . Accordingly, the concentration of soluble heme in the periplasm was $3.76 \mathrm{~mm}$. Of note, the total heme concentration of the periplasm will be even higher, as a number of heme proteins like CymA, MtrA or DmsE are membrane bound.

Interestingly, $S$. oneidensis produces a core pattern of different $C$-type cytochromes irrespective of the anoxic electron acceptor. As depicted in Figure 1, this held true even under conditions where the electron transport chain to the TEA was $C$-typecytochrome-independent, as thiosulfate reduction is catalyzed by the psrABC gene product. PsrA is a molybdoprotein; PsrB is an iron-sulfur-protein; and PsrC is the membrane anchor. The only cytochrome

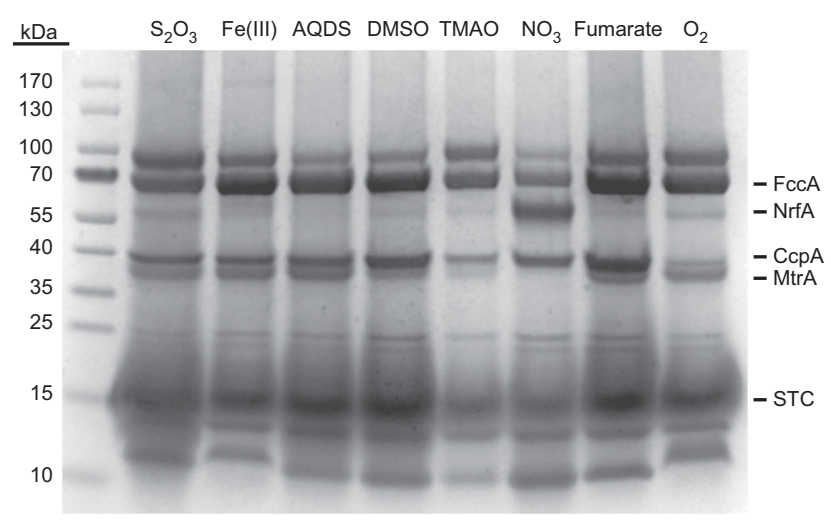

Figure 1 Heme Stain of periplasmic fractions of $S$. oneidensis MR-1. S. oneidensis was grown in M4 media with different anaerobic terminal electron acceptors as well as oxygen. With the exception of $\mathrm{NO}_{3}^{-}$, a constant pattern of expressed $c$-type cytochromes was detected under all tested conditions. Even aerobically grown cells in late logarithmic phase seem to produce the same set of $c$-type cytochromes. Corresponding bands to the $\mathrm{NO}_{2}^{-}$reductase NrfA, the fumarate reductase FccA, Ccpa, MtrA and STC are labeled.

protein that was strongly upregulated under one specific condition was the nitrite reductase NrfA, whose production was previously shown to depend primarily on the conditions of nitrate ammonification (Gao et al., 2009). NrfA identification was performed by mass spectrometric analysis. Nevertheless, a corresponding, but less apparent, heme-containing protein band was also visible under all other conditions tested. Also present under all conditions, but in major quantities, were the fumarate reductase FccA and the small tetraheme cytochrome (STC). Of note, the depicted results were also obtained if not only one transfer from oxic lysogeny broth media to anoxic minimal media with an excess concentration of lactate was conducted but also after four individual anoxic transfers of the cultures with balanced concentrations of lactate regarding the capacity of the added electron acceptors (Supplementary Figure S3).

S. oneidensis contains an electron transfer network and storage compartment

It is not possible to concentrate periplasmic fractions under laboratory conditions to a heme content of $3.76 \mathrm{~mm}$ or more owing to protein precipitation. Hence, it can be assumed that the periplasm is a conductive, highly viscous matrix that bridges the gap between the inner and outer membrane. Furthermore, the heme-containing components seem to be produced following an electron acceptor-independent regulatory mechanism. Nevertheless, it has been assumed from knockout studies that only a limited number of specific cytochromes is necessary for establishing the individual electron transport chains. Therefore, the relevance of a surplus production of $c$-type cytochromes for the ecological fitness of the organism remains enigmatic.

One hypothesis is that these cytochromes comprise an electron capacitor that allows for oxidation 
of a carbon source, irrespective of the presence of an electron acceptor (Esteve-Nunez et al., 2008). A corresponding hypothesis would be that $S$. oneidensis follows the ecological strategy of a generalist, using existing resources faster than competing organisms. The simultaneous expression of a multitude of electron transport chains would be one possible way to enable such a metabolic program. The following experiments were conducted to analyze whether the expressed $C$-type cytochromes are indeed part of functional electron transport chains to various electron acceptors and to determine whether electrons stored within the cytochrome pool can be released to these electron acceptors irrespective of preceding growth conditions. S. oneidensis cells were grown on ferric iron, harvested during logarithmic growth and washed in an electron acceptor-free medium containing lactate. This led to a complete reduction of the cytochrome pool as detected using a comparison of spectra from the cell suspensions with further dithionite-reduced samples. The cells were then washed in medium without a donor or acceptor, and different anoxic electron acceptors were added to the cell suspensions. As indicated in Figure 2a, the cells were reoxidized after the addition of all of the electron acceptors tested with the exception of thiosulfate, which like the addition of buffer, did not change the redox status of the cytochrome pool. Nevertheless, electron acceptors spanning a redox potential window between - $184 \mathrm{mV}$ (anthraquinone-2,6-disulfonate) and $+433 \mathrm{mV}\left(\mathrm{NO}_{3}^{-}\right)$led to complete oxidation, indicating not only the presence of functional electron transport chains, but furthermore, their interconnection. As an example, MtrC, MtrA and OmcA are not only involved in the reduction of TMAO, fumarate, $\mathrm{NO}_{3}^{-}$and DMSO as terminal reductases, but also apparently can be reoxidized via reverse flow of electrons towards these alternative electron acceptors.

Interestingly, the same behavior of complete reoxidation (with the same exception of thiosulfate) could be observed if aerobically grown cells were washed and prior to the experiment placed for $3 \mathrm{~h}$ in an anoxic environment with lactate but without any TEA (data not shown). From the multitude of electron acceptors that $S$. oneidensis can use, environmental ferric iron forms probably have the lowest redox potential. If the parallel expression of interconnected electron transport chains under anoxic conditions is a general strategy of the organism, one would predict that the same behavior should also be detectable under nitrate-reducing conditions. In agreement with this hypothesis, reduced nitrategrown cells could be reoxidized by the addition of nitrate, fumarate, anthraquinone-2,6-disulfonate and ferric citrate (data not shown). Nevertheless, the addition of DMSO or TMAO only let to a slight reoxidation of the cytochrome pool, if at all.

Multidimensional protein identification technology was utilized to confirm the presence of

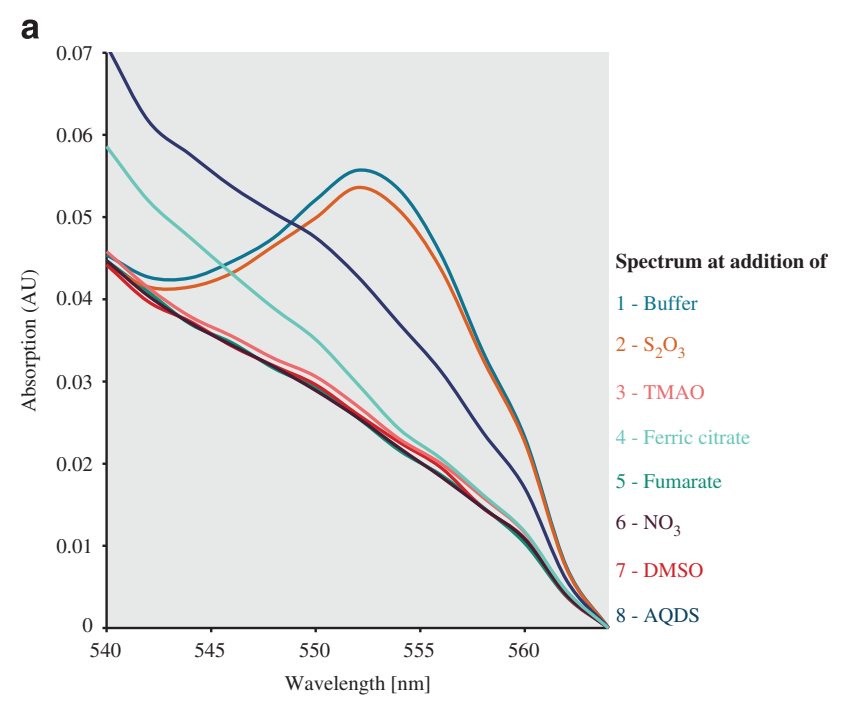

beriplasm \{ \begin{tabular}{ccccccc} 
UniProt & Locus tag & Name & Substrate & Score & Peptides & Hits \\
\hline P83223 & 970 & FccA & Fumarate & 442.83 & 25 & 497 \\
Q8EF24 & 2178 & CcpA & $\mathrm{H}_{2} \mathrm{O}_{2}$ & 194.74 & 15 & 61 \\
Q8EIJ1 & 848 & NapA & Nitrate & 8.76 & 1 & 1 \\
Q8EAC7 & 3980 & NrfA & Nitrite & 10.9 & 1 & 1 \\
Q8EG34 & 1777 & MtrC & Iron & 32.28 & 3 & 3 \\
Q8EH03 & 1429 & DmsA & DMSO & 70.81 & 7 & 9 \\
Q8EH02 & 1430 & DmsB & DMSO & 33.79 & 2 & 5 \\
Q8E8S0 & 4591 & CymA & - & 28.31 & 2 & 6
\end{tabular}

Figure 2 Electron transferring network. (a) Ferric iron-grown cells were lactate reduced and subjected to different terminal electron acceptors. Their redox state was monitored via absorption spectroscopy. A reduced state is indicated by an absorption peak at $552 \mathrm{~nm}$, whereas the peak is missing in the oxidized state. As depicted, the cells were able to discharge their electron cargo onto all electron acceptors applied excepting $\mathrm{S}_{2} \mathrm{O}_{3}^{-}$. (b) Summary of terminal reductases identified via MudPIT analysis of periplasmic and membrane fractions of ferric iron-grown $S$. oneidensis cells.

multiple terminal reductases in the ferric irongrown cells. Enzymes for the reduction of nitrate, nitrite, DMSO, fumarate, ferric iron, and hydrogen peroxide were detectable (Figure 2b). Interestingly, although a TMAO-dependent reoxidation was observed in the ferric iron-grown cells, peptides corresponding to the TMAO reductase TorA were not identified. Nevertheless, several DMSO reductases can also reduce TMAO (Dos Santos et al., 1998). Hence, TMAO-dependent reoxidation might be due to an unspecificity of the DMSO reductase.

\section{FCCA and STC as prominent and important} cytochromes with overlapping activity

The two periplasmic cytochromes FccA and STC were highly transcribed under all anaerobic growth conditions tested, and they were thus identified as potential electron-transfer hubs, interconnecting the individual electron transport chains. Hence, a markerless double-deletion mutant for the corresponding genes was constructed and tested for possible growth deficiencies under anoxic conditions. 
The double mutant showed a strong phenotype when tested under the conditions of ferric iron, DMSO and nitrate reduction. It had an extremely elongated, but reproducible, lag-phase under ferric iron-reducing conditions, after which it grew to densities similar to those of wild-type (Figure 3a). After this lag-phase, it produced a different pattern of periplasmic cytochromes (Supplementary Figure S2), suggesting that the mutant was functioning under a different regulatory protocol. It is not yet clear how this new mixture of heme-containing proteins rescued the mutant phenotype. Nevertheless, the cct $A / f_{c c} A$ deletion mutant was unable to grow on DMSO and initially showed minor growth on nitrate, but stopped after reaching $20 \%$ of the wild-type yield (Figures $3 \mathrm{~b}$ and c). In contrast, no phenotype was detectable for anoxic growth with TMAO as well as under oxic growth conditions. All growth deficiencies could be rescued via the expression of either $\operatorname{cct} A$ or $f c c A$ from an inducible plasmid, indicating the overlapping activity of the two cytochromes (Figure 3).

Transcriptomic analysis of the $\operatorname{cct} A / f_{c c} A$ double mutant

The surprising severity of the $\operatorname{cct} A$ and $f c c A$ double deletion could be either the result of an inability to transport electrons through the periplasm or to regulate the metabolism according to anoxic conditions. For instance, the latter results from a deletion of the $\operatorname{crp}$ gene, as the corresponding protein is central for cyclic AMP-coordinated regulation of the shift from an aerobic to anaerobic metabolism (Saffarini et al., 2003). The crp mutant cannot grow anaerobically with DMSO, ferric iron, or fumarate as electron acceptors, which is similar to the observed phenotype of the $c c t A / f_{C C A}$ mutant.

Owing to the similarity of the $\operatorname{crp}$ and $\operatorname{cct} A / f_{c c} A$ mutant phenotypes, it was hypothesized that in addition to their role in electron transport, the two cytochromes might have an additional function in the regulation of anaerobic metabolism.
Therefore, the transcriptomes of the double-deletion mutant and wild-type were compared during the shift from aerobic to anaerobic growth with ferric iron and evaluated in relation to a $c r p$ - or $c y a C$ deletion mutation (Table 1). CyaC is the major cyclic adenosine monophosphate adenylate cyclase and therefore involved in the synthesis of the effector molecule (Charania et al., 2009). Surprisingly, the $\operatorname{cct} A / f_{C C} A$-deletion mutant was not affected in its transcription of essential $C$-type cytochromes such as $\operatorname{mtr} C, n r f A$ or $c y m A$. The corresponding genes for DMSO and nitrate reductase were significantly upregulated. The expression of known components of heme and menaquinone biosynthesis was not negatively affected. Concerning the central carbon metabolism, a slight upregulation of lactate dehydrogenase genes was detected. An obvious similarity to $\operatorname{crp}$ and $c y a C$ mutants was the downregulation of the pyruvate formate lyase. Moreover, the mutation led to a downregulation of the pta and ackA genes, which catalyze the ATP-yielding conversion of acetyl-CoA to acetate. A complete summary of the results from transcriptomic analysis can be found in Data S1.

Nitrate and nitrite reduction in cell suspensions It is possible that STC and FCcA have a central role as an electron transfer hub providing the electron transfer chains to DMSO, ferric citrate and nitrate with CymA-based electrons and/or have a regulatory function that causes a decrease in lactate metabolism via the downregulation of $p f l$, ackA and $p t a$. Both functions could explain the phenotype under anoxic conditions.

The last set of experiments was conducted to estimate the impact of the $p f l, a c k A$ and $p t a$ downregulation on the mutant phenotype. It is known that NrfA directly interacts with CymA (Gao et al., 2009). Therefore, S. oneidensis wildtype and double-mutant strains were grown under oxic conditions, then washed and incubated under anoxic conditions to induce the expression of genes a

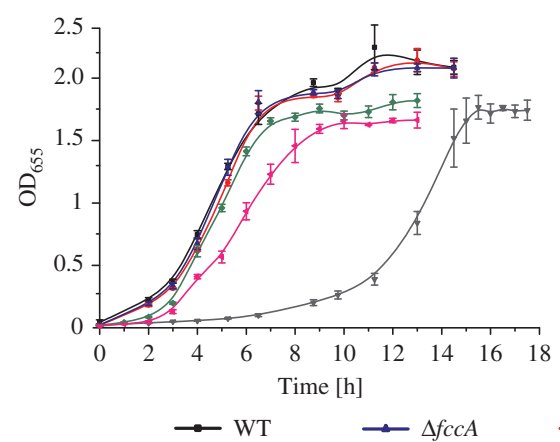

b

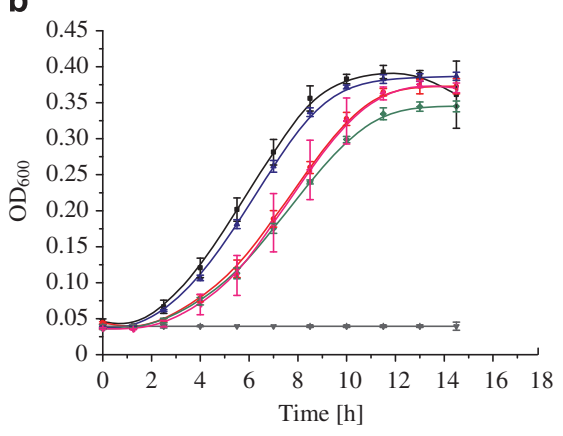

C

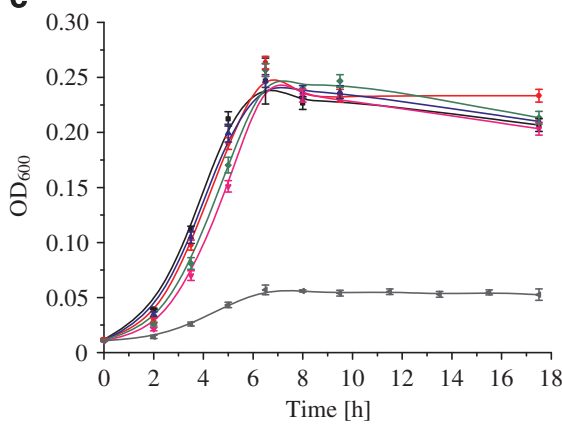

Figure 3 Growth of $S$. oneidensis wild-type and mutants on different terminal electron acceptors. The panels show growth monitored by optical density on (a) ferric citrate; (b) DMSO; (c) $\mathrm{NaNO}_{3}$. WT (JG7); $\Delta s t c$ (JG441); $\Delta f_{C c} A$ (JG207); $\Delta s t c \Delta f_{C c} A$ (JG684); $\Delta s t c \Delta f_{C c} A$ pBAD_stc (JG700); $\Delta s t c \Delta f c c A$ pBAD_fccA (JG837). JG684 shows growth deficiencies on ferric iron, DMSO and NaNO ${ }_{3}$. Growth curves represent at least three independent replicates, error bars indicate standard deviation. 
Table 1 Transcriptomic response of $\Delta c c t A / f_{c c} A$ compared with the wild-type during the initial transition from oxic to anoxic growth with ferric iron as electron acceptor

\begin{tabular}{|c|c|c|c|c|c|}
\hline & SO_number & Gene name & Fold change & $\Delta c r p$ & $\triangle c y a C$ \\
\hline \multirow[t]{7}{*}{ Lactate metabolism } & SO_1518 & $\operatorname{lld} G$ & 2.7 & & \\
\hline & SO_1519 & $l l d F$ & 2.6 & & \\
\hline & SO_1520 & $\operatorname{lld} E$ & 2.4 & & \\
\hline & SO 2912 & $p f l B$ & -3.0 & $\downarrow$ & $\downarrow$ \\
\hline & SO_2913 & pflA & -3.0 & $\downarrow$ & $\downarrow$ \\
\hline & SO_2915 & $\operatorname{ack} A$ & -3.8 & $\downarrow$ & $\downarrow$ \\
\hline & SO 2916 & pta & -3.6 & $\downarrow$ & $\downarrow$ \\
\hline \multirow[t]{2}{*}{ Heme biosynthesis } & SO_0027 & hemG-2 & 2.2 & $\downarrow$ & \\
\hline & SO 3720 & hem $G-3$ & 2.3 & $\downarrow$ & \\
\hline \multirow[t]{5}{*}{ Respiratory chains } & SO_0845 & nарB & 6.4 & $\downarrow$ & \\
\hline & SO_0848 & nap $A$ & 10.3 & $\downarrow$ & \\
\hline & SO 1428 & $d m s F$ & 5.8 & $\downarrow$ & $\downarrow$ \\
\hline & SO_1429 & $d m s A$ & 6.0 & $\downarrow$ & $\downarrow$ \\
\hline & SO_1430 & $d m s B$ & 6.2 & $\downarrow$ & $\downarrow$ \\
\hline
\end{tabular}

Indicated are the significant fold changes in expression of genes corresponding to proteins involved in oxic/anoxic regulation, heme and menaquinone biosynthesis, lactate metabolism as well as anaerobic respiratory chains. Variations in expression were regarded as not significantly altered (NS) if the fold change was below 2 or the padj value was above 0.05 . The expression data is compared with a study conducted by Charania et al. (2009) that described the impact of crp or cyaC deletion on gene expression under oxygen limiting conditions compared with the wild type. Arrows indicate up $(\uparrow)$ or down $(\downarrow)$ regulation of the corresponding genes in the respective mutant strains.

involved in anaerobic electron transfer. The gene for the nitrite reductase NrfA was additionally expressed from an inducible plasmid in both cell types. The resulting cell suspensions were tested for their ability to reduce either nitrate or nitrite. A decrease in nitrite and nitrate reduction should be detectable if central metabolic conversion rates, and hence electron supply, are limiting. In contrast, if the central metabolism is not limiting electron transfer, then the two cell types should reduce nitrite at similar rates (NrfA is directly reduced by CymA), and nitrate reduction should be severely inhibited.

As indicated in Figure 4, both the wild-type and double-deletion mutant strains reduced nitrite at equal rates. The mutant was unable to transport electrons to nitrate, even though the expression of the nitrate reductase NapA was upregulated. Therefore, electron transport was not only limited by the conversion of lactate, but also by a loss of the important and necessary periplasmic electron transferring proteins STC and FccA.

\section{Discussion}

The periplasm of $S$. oneidensis is a reaction room that is filled with a high concentration of $c$-type cytochromes. These proteins have a central function in anoxic electron transfer to a multitude of different electron acceptors. Several researchers already showed a coexpression of several $c$-type cytochromes at the level of the transcriptome (Beliaev et al., 2005; Gralnick et al., 2005). Nevertheless, it is unknown what this coexpression really infers regarding the physiology of the organism and the biochemistry of electron transfer to the arsenal of electron acceptors that $S$. oneidensis is able to reduce.

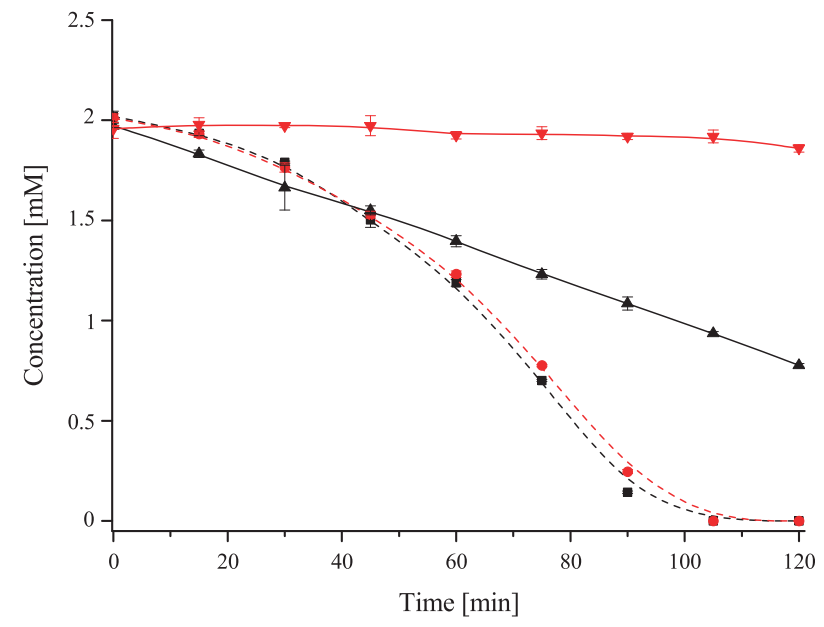

Figure $4 \quad \mathrm{NO}_{3}^{-}$and $\mathrm{NO}_{2}^{-}$reduction. $S$. oneidensis wild-type (WT) and $\Delta s t c \Delta f_{C c} A$ mutant (JG684) cell suspensions were tested for $\mathrm{NO}_{3}^{-}$and $\mathrm{NO}_{2}^{-}$reduction, respectively. Nitrate consumption by wild-type cells is indicated by a black solid line. Cell suspensions of $\Delta s t c \Delta f_{C c} A$ show no nitrate-reducing activity (red solid line). Cell suspensions of both, wild-type (black striped line) and $\Delta s t c \Delta f_{C c} A$ mutant (red striped line) show nitrite reduction with highly similar rates, indicating that the central metabolism is not affected by the deletion of stc and $f_{C C A}$, as the nitrite reductase NrfA is directly coupled to CymA. Error bars represent standard deviation of three independent replicates.

A single cell contains almost 700000 heme sites that could be used as a repository for electron storage, which to some extent enables the electron acceptorindependent oxidation of an available carbon source. For instance, because $S$. oneidensis releases four electrons during the oxidation of lactate to acetate and $\mathrm{CO}_{2}, 175000$ lactate molecules could be oxidized by a $c$-type cytochrome pool of 700000 hemes. Consequently, at least 175000 ATP molecules would be produced during the substrate level phosphorylation reaction from acetyl-CoA to acetate. 
The heme sites within the cytochrome pool are interconnected and therefore, addition of an electron acceptor to cells where electron storage sites are fully occupied leads to a complete reoxidation of the $c$-type cytochrome pool. Of note, a variety of different electron acceptors leads to a similar total reoxidation of the pool. A clear hierarchical expression dependent on the redox potential of the TEA is lacking. For instance, the cells produce the components of the extended respiratory chain to the cell surface, even when they are pre-grown with nitrate.

This regulatory mechanism, which is independent of the redox potential, is also mirrored in the strategy of respiratory periplasmic branching initiated by the cytoplasmic membrane protein CymA. The electron transfer chains to DMSO (DMSO/DMS: $\quad+160 \mathrm{mV})$, nitrate $\left(\mathrm{NO}_{3}^{-} / \mathrm{NO}_{2}^{-}\right.$: $+433 \mathrm{mV})$, nitrite $\left(\mathrm{NO}_{2}^{-} / \mathrm{NH}_{4}^{+}:+340 \mathrm{mV}\right)$, ferric iron $(+372$ to $-177 \mathrm{mV})$, manganese oxide $\left(\mathrm{Mn}^{4+} / \mathrm{Mn}^{2+}:+612\right.$ to $\left.+269 \mathrm{mV}\right)$ and fumarate (fumarate/succinate: $+33 \mathrm{mV}$ ) all rely on this menaquinol oxidase (Thauer et al., 1977; Straub et al., 2001). This might be an adaptation to environments such as sediments in which rapid redox cycling occurs. Interestingly, this indeed seems to be the niche of the genus Shewanella, as most of the isolates cultivated thus far originate from sediment samples or redox-stratified environments (Fredrickson et al., 2008). Of note, experiments conducted by Esteve-Nunez et al. (2008), showed that Geobacter sulfurreducens, another model organisms for extracellular respiration, growing on ferric iron was unable to release the electrons of the cytochrome pool to fumarate. This observation is in keeping with the characteristics of G. sulfurreducens cells, a specialist for anoxic growth.

The concept of transient electron storage is to some extent corroborated by results from Harris et al. (2012). The authors observed a distinct response called electrokinesis when $S$. oneidensis cells were in the vicinity of an insoluble electron acceptor in the absence of oxygen. Cells that made an initial contact with the electron acceptor showed an increased swimming speed with a high rate of flagella reversal that kept the cells in close proximity to the electron acceptor. Upon following collisions with the electron acceptor, the cells either attached to the material or began the same program again. In other words, the periodic discharge of catabolic electrons seems to sustain the energy metabolism of the cell. Transient electron storage could provide a tool to maintain a constant metabolic flux, and thus ATP yield, even if the cells are disconnected from the respiratory electron acceptor. Of note, it is not clear yet whether $c$-type cytochromes are the only electron storage entity in $S$. oneidensis or whether other redox-active compounds also contribute.

Interestingly, although the electron transport pathways to several electron acceptors seem to be overlapping and coexpressed at least to some extent, it could be shown that $S$. oneidensis has a specific chemotactic behavior (Nealson et al., 1995). For instance, nitrate grown cells move along a nitrate gradient even in the presence of fumarate. Nevertheless, addition of DMSO blocks this chemotactic behavior. It will be part of future studies to analyze interconnection between electron transfer kinetics and chemotactic behavior.

\section{STC and FCCA are the major constituents of the} periplasmic c-type cytochrome pool

So far, several models were postulated regarding periplasmic electron transfer in S. oneidensis. For instance, in this journal Gao et al. (2009) assumed that electron transfer between CymA and NapAB is direct and does not involve a further electron shuttling protein. Other researchers raised the hypothesis that CymA would form electron transfer complexes with the outer membrane anchored terminal reductases MtrABC or DmsABEF (Ross et al., 2011; McMillan et al., 2012). Nevertheless, this study reveals the necessity of at least one connecting protein, either FccA or STC for coupling respiratory oxidation of CymA to effective electron transfer to at least ferric citrate, DMSO and nitrate. Our results are consistent with previous work from Fonseca et al. (2013). The authors elucidated a number of interactions for STC and FccA with other periplasmic cytochromes, including MtrA and CymA. Interestingly, the two proteins cannot interact with each other, which might be a consequence of their negative surface charge. The binding constants reported by the authors favor weak transient interactions for FCCA and STC with CymA or MtrA, which is typical for transient electron-transfer interactions. The high concentrations of these proteins in the periplasm would probably allow for stochastic, but highly frequent, interactions with other parts of the individual electron transfer chains. As these are interconnected, it seems likely that only the presence of a certain electron acceptor triggers the electron flow into one particular reaction. Still, the question of whether periplasmic electron transfer follows weak interactions or the formation of static protein complexes is still under debate, because McMillan et al. (2013) observed CymA-triggered absorption of FccA onto lipid vesicles in an in vitro system. Thus far, what this experimental result infers for the in vivo situation remains unresolved.

In conclusion, we introduced a novel microbial metabolic strategy, which potentially results in a fitness benefit under conditions of rapid redox cycling. The simultaneous production of different functional respiratory chains is sustained by the production of the central electron transfer hubs, STC and FccA. The high concentration of these proteins in the periplasm could not only result in the formation of an electron capacitor, but it also 
ensures an equal distribution of respiratory electrons throughout the periplasm and onto different electron transfer chains. This function as a distributor cannot be adopted only by CymA, because its membrane anchor does not allow for rapid interactions with the total available pool of soluble terminal reductases.

\section{Conflict of Interest}

The authors declare no conflict of interest.

\section{Acknowledgements}

Thanks to Nico Bursac and André Dittrich for help in modeling the periplasmic space. We are grateful for financial support from the German Research Foundation (DFG).

\section{References}

Anders S, Huber W. (2010). Differential expression analysis for sequence count data. Genome Biol 11: R106.

Beliaev AS, Klingeman DM, Klappenbach JA, Wu L, Romine MF, Tiedje JM et al. (2005). Global transcriptome analysis of Shewanella oneidensis MR-1 exposed to different terminal electron acceptors. J Bacteriol 187: 7138-7145.

Berry EA, Trumpower BL. (1987). Simultaneous determination of hemes $a, b$, and $c$ from pyridine hemochrome spectra. Anal Biochem 161: 1-15.

Breuer M, Rosso KM, Blumberger J, Butt JN. (2014). Multihaem cytochromes in Shewanella oneidensis MR-1: structures, functions and opportunities. $J R$ Soc Interface 12: 20141117.

Charania MA, Brockman KL, Zhang Y, Banerjee A, Pinchuk GE, Fredrickson JK et al. (2009). Involvement of a membrane-bound class III adenylate cyclase in regulation of anaerobic respiration in Shewanella oneidensis MR-1. J Bacteriol 191: 4298-4306.

Dohnalkova AC, Marshall MJ, Arey BW, Williams KH, Buck EC, Fredrickson JK. (2011). Imaging hydrated microbial extracellular polymers: comparative analysis by electron microscopy. Appl Environ Microbiol 77: 1254-1262.

Dos Santos JP, Iobbi-Nivol C, Couillault C, Giordano G, Mejean V. (1998). Molecular analysis of the trimethylamine N-oxide (TMAO) reductase respiratory system from a Shewanella species. J Mol Biol 284: 421-433.

Doetsch A, Eckweiler D, Schniederjans M, Zimmermann A, Jensen V, Scharfe M et al. (2012). The Pseudomonas aeruginosa transcriptome in planktonic cultures and static biofilms using RNA sequencing. PloS One 7: e3109210.1371/journal.pone.0031092.

Esteve-Nunez A, Sosnik J, Visconti P, Lovley DR. (2008). Fluorescent properties of $c$-type cytochromes reveal their potential role as an extracytoplasmic electron sink in Geobacter sulfurreducens. Environ Microbiol 10: $497-505$.

Fonseca BM, Paquete CM, Neto SE, Pacheco I, Soares CM, Louro RO. (2013). Mind the gap: cytochrome interactions reveal electron pathways across the periplasm of Shewanella oneidensis MR-1. Biochem J449: 101-108.
Fredrickson JK, Romine MF, Beliaev AS, Auchtung JM, Driscoll ME, Gardner TS et al. (2008). Towards environmental systems biology of Shewanella. Nat Rev Microbiol 6: 592-603.

Gao H, Barua S, Liang Y, Wu L, Dong Y, Reed S et al. (2010). Impacts of Shewanella oneidensis $c$-type cytochromes on aerobic and anaerobic respiration. Microb Biotech 3: 455-466.

Gao HC, Yang ZK, Barua S, Reed SB, Romine MF, Nealson KH et al. (2009). Reduction of nitrate in Shewanella oneidensis depends on atypical NAP and NRF systems with NapB as a preferred electron transport protein from CymA to NapA. ISME J 3: 966-976.

Gibson DG, Young L, Chuang R-Y, Venter JC, Hutchison CA, Smith HO. (2009). Enzymatic assembly of DNA molecules up to several hundred kilobases. Nat Methods 6: 343-345.

Goh EB, Bledsoe PJ, Chen LL, Gyaneshwar P, Stewart V, Igo MM. (2005). Hierarchical control of anaerobic gene expression in Escherichia coli K-12: the nitrateresponsive NarX-NarL regulatory system represses synthesis of the fumarate-responsive DcuS-DcuR regulatory system. J Bacteriol 187: 4890-4899.

Gordon EH, Pike AD, Hill AE, Cuthbertson PM, Chapman SK, Reid GA. (2000). Identification and characterization of a novel cytochrome c(3) from Shewanella frigidimarina that is involved in Fe(III) respiration. Biochem $J$ 349: 153-158.

Gralnick JA, Brown CT, Newman DK. (2005). Anaerobic regulation by an atypical Arc system in Shewanella oneidensis. Mol Microbiol 56: 1347-1357.

Green J, Guest JR. (1993). A role for iron in transcriptional activation by FNR. FEBS Lett 329: 55-58.

Harris HW, El-Naggar MY, Nealson KH. (2012). Shewanella oneidensis MR-1 chemotaxis proteins and electron-transport chain components essential for congregation near insoluble electron acceptors. Biochem Soc Trans 40: 1167-1177.

Hartshorne RS, Reardon CL, Ross D, Nuester J, Clarke TA, Gates AJ et al. (2009). Characterization of an electron conduit between bacteria and the extracellular environment. Proc Natl Acad Sci USA 106: 22169-22174.

Langmead B, Salzberg SL. (2012). Fast gapped-read alignment with Bowtie 2. Nat Methods 9: 357-359.

Marritt SJ, Lowe TG, Bye J, McMillan DG, Shi L, Fredrickson J et al. (2012). A functional description of CymA, an electron-transfer hub supporting anaerobic respiratory flexibility in Shewanella. Biochem $J$ 444: 465-474.

McMillan DG, Marritt SJ, Firer-Sherwood MA, Shi L, Richardson DJ, Evans SD et al. (2013). Protein-protein interaction regulates the direction of catalysis and electron transfer in a redox enzyme complex. J Amer Chem Soc 135: 10550-10556.

McMillan DGG, Marritt SJ, Butt JN, Jeuken LJC. (2012). Menaquinone-7 is specific cofactor in tetraheme quinol dehydrogenase CymA. J Biol Chem 287: 14215-14225.

Meyer TE, Tsapin AI, Vandenberghe I, de Smet L, Frishman D, Nealson KH et al. (2004). Identification of 42 possible cytochrome c genes in the Shewanella oneidensis genome and characterization of six soluble cytochromes. OMICS 8: 57-77.

Myers CR, Myers JM. (1997). Cloning and sequence of суmA a gene encoding a tetraheme cytochrome $c$ required for reduction of iron(III), fumarate, and nitrate by Shewanella putrefaciens MR-1. J Bacteriol 179: 1143-1152. 
Nealson KH, Moser DP, Saffarini DA. (1995). Anaerobic electron acceptor chemotaxis in Shewanella putrefaciens. Appl Environ Microbiol 61: 1551-1554.

Parkhomchuk D, Borodina T, Amstislavskiy V, Banaru M, Hallen L, Krobitsch S et al. (2009). Transcriptome analysis by strand-specific sequencing of complementary DNA. Nucleic Acids Res 37: e123.

Richardson DJ, Edwards MJ, White GF, Baiden N, Hartshorne RS, Fredrickson J et al. (2012). Exploring the biochemistry at the extracellular redox frontier of bacterial mineral Fe(III) respiration. Biochem Soc Trans 40: 493-500.

Romine MF, Carlson TS, Norbeck AD, McCue LA, Lipton MS. (2008). Identification of mobile elements and pseudogenes in the Shewanella oneidensis MR-1 genome. Appl Environ Microbiol 74: 3257-3265.

Ross DE, Ruebush SS, Brantley SL, Hartshorne RS, Clarke TA, Richardson DJ et al. (2007). Characterization of protein-protein interactions involved in iron reduction by Shewanella oneidensis MR-1. Appl Environ Microbiol 73: 5797-5808.

Ross DE, Flynn JM, Baron DB, Gralnick JA, Bond DR. (2011). Towards electrosynthesis in Shewanella: energetics of reversing the Mtr pathway for reductive metabolism. PloS One 6: e16649.

Saffarini DA, Schultz R, Beliaev A. (2003). Involvement of cyclic AMP (cAMP) and cAMP receptor protein in anaerobic respiration of Shewanella oneidensis. J Bacteriol 185: 3668-3671.

Schicklberger M, Bücking C, Schuetz B, Heide H, Gescher J. (2011). Involvement of the Shewanella oneidensis decaheme cytochrome MtrA in the periplasmic stability of the $\beta$-barrel protein MtrB. Appl Environ Microbiol 77: 1520-1523.

Schicklberger M, Sturm G, Gescher J. (2013). Genomic plasticity enables a secondary electron transport pathway in Shewanella oneidensis. Appl Environ Microbiol 79: 1150-1159.

Schuetz B, Schicklberger M, Kuermann J, Spormann AM, Gescher J. (2009). Periplasmic electron transfer via the $c$-type cytochromes MtrA and FccA of Shewanella oneidensis MR-1. Appl Environ Microbiol 75: 7789-7796.
Schwalb C, Chapman SK, Reid GA. (2002). The membrane-bound tetrahaem $c$-type cytochrome CymA interacts directly with the soluble fumarate reductase in Shewanella. Biochem Soc Trans 30: 658-662.

Shanks RM, Caiazza NC, Hinsa SM, Toutain CM, O’Toole GA. (2006). Saccharomyces cerevisiae-based molecular tool kit for manipulation of genes from Gram-negative bacteria. Appl Environ Microbiol 72: 5027-5036.

Shi L, Squier TC, Zachara JM, Fredrickson JK. (2007). Respiration of metal (hydr)oxides by Shewanella and Geobacter: a key role for multihaem $c$-type cytochromes. Mol Microbiol 65: 12-20.

Spiro S, Guest JR. (1990). FNR and its role in oxygenregulated gene expression in Escherichia coli. FEMS Microbiol Rev 6: 399-428.

Straub KL, Benz M, Schink B. (2001). Iron metabolism in anoxic environments at near neutral pH. FEMS Microbiol Ecol 34: 181-186.

Thauer RK, Jungermann K, Decker K. (1977). Energy conservation in chemotrophic anaerobic bacteria. Bacteriol Rev 41: 100-180.

Thomas PE, Ryan D, Levin W. (1976). An improved staining procedure for the detection of the peroxidase activity of cytochrome P-450 on sodium dodecyl sulfate polyacrylamide gels. Anal Biochem $\mathbf{7 5}$ : 168-176.

Tsapin AI, Vandenberghe I, Nealson KH, Scott JH, Meyer TE, Cusanovich MA et al. (2001). Identification of a small tetraheme cytochrome c and a flavocytochrome c as two of the principal soluble cytochromes $\mathrm{c}$ in Shewanella oneidensis strain MR1. Appl Environ Microbiol 67: 3236-3244.

Unden G, Duchene A. (1987). On the role of cyclic AMP and the Fnr protein in Escherichia coli growing anaerobically. Arch Microbiol 147: 195-200.

Warnes GR, Bolker B, Bonebakker L, Gentleman R, Huber W, Liaw A et al. (2013). gplots: Various R programming tools for plotting data. From: WWW URL http://CRAN. R-project.org/package=gplots.

Supplementary Information accompanies this paper on The ISME Journal website (http://www.nature.com/ismej) 